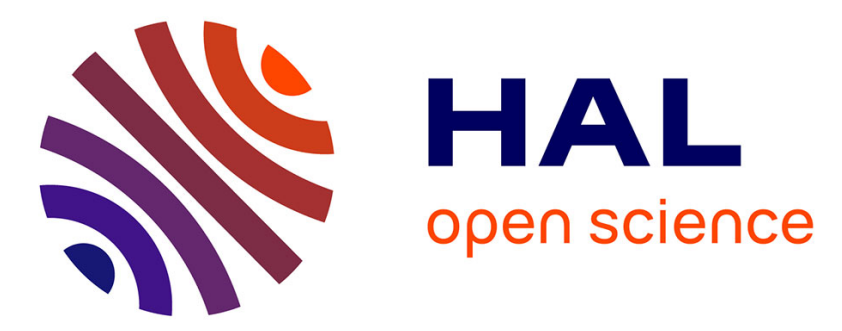

\title{
Online temporal reasoning for event and data streams processing
}

Jean-Philippe Poli, Laurence Boudet, David Mercier

\section{To cite this version:}

Jean-Philippe Poli, Laurence Boudet, David Mercier. Online temporal reasoning for event and data streams processing. 2016 IEEE International Conference on Fuzzy Systems (FUZZ-IEEE), Jul 2016, Vancouver, Canada. pp.7737974, 10.1109/FUZZ-IEEE.2016.7737974 . hal-01890333

\section{HAL Id: hal-01890333 https://hal.science/hal-01890333}

Submitted on 26 Feb 2022

HAL is a multi-disciplinary open access archive for the deposit and dissemination of scientific research documents, whether they are published or not. The documents may come from teaching and research institutions in France or abroad, or from public or private research centers.
L'archive ouverte pluridisciplinaire $\mathbf{H A L}$, est destinée au dépôt et à la diffusion de documents scientifiques de niveau recherche, publiés ou non, émanant des établissements d'enseignement et de recherche français ou étrangers, des laboratoires publics ou privés. 


\title{
Online Temporal Reasoning For Event And Data Streams Processing
}

\author{
Jean-Philippe Poli, Laurence Boudet, David Mercier \\ CEA, LIST, Data Analysis and System Intelligence Laboratory, \\ 91191 Gif-sur-Yvette cedex, France. \\ Email: firstname.lastname@cea.fr
}

February 25, 2022

\begin{abstract}
Online fuzzy expert systems can be used to process data and event streams, providing a powerful way to handle their uncertainties and their inaccuracy. Moreover, human experts can decide how to process the streams with rules close to natural language. However, to extract high level information from these streams, they need at least to describe the temporal relations between the data or the events.

In this paper, we propose a straightforward way to design temporal operators which relies on the mathematical definition of some base operators and then their combination into more sophisticated operators to assess precedence, periodicity or persistence. We also introduce the concept of expiration of temporal expressions on online fuzzy expert systems, that is to say the capacity to change the values of outputs whereas the inputs have not changed.
\end{abstract}

\section{Introduction}

The emergence of connected objects and of the Internet of Things is leading towards a continuous data acquisition from different devices and sensors. We focus in this paper on information streams produced by a device network, i.e. couples $(t, d)$ where $t$ is a timestamp and $d$ is data. Sensors may produce either measurements at a constant or a dynamic sampling rate (data streams, e.g. thermometers), or events when they occur (event streams, e.g. presence sensors). Information flow processing mainly consists in filtering, gathering and combining those events in order to build a higher level information [1] and is closely related to pattern and scenario recognition [2].

A scenario, or pattern or chronicle, can be defined [3] [4] as a sequence of events and a set of temporal constraints between them. Many authors [5] [6] [7] [8] thus claim that temporal operators are the minimum requirement to describe scenarios or complex events. For instance, in classical logic, Allen relations [9] 
have lead to successful applications [10] [11] [12] of scenario recognition. More recently, Dousson [13] distinguished two families of scenarios recognition algorithms: Store Totally Recognized Scenarios (STRS) strategy which aims at analyzing only past events and Store Partially Recognized Scenarios (SPRS) strategy which is able to prospect for upcoming events. Both of the strategies suffer from different drawbacks: STRS strategy must store all the previously recognized scenarios and SPRS strategy implies that scenarios have to be bounded in time and must maintain all the possible partial scenarios. Other approaches for scenario recognition use graphical models (e.g. finite state automatons or Petri nets) [14], event calculus or even machine learning algorithms [4]: although these methods are powerful, they are not intuitive and require an expert level to handle them. Finally, expert systems based on boolean logic have been combined with event detection algorithms in order to use event-condition-action rules for recognizing patterns [15]. All these methods differ by the way the events and the constraints are described.

Whatever the type of items in the streams, i.e. either data or events, the information may be incomplete and imprecise by nature [16]. For instance, sensors may be out of order or inaccurate, or data may be noisy. For the last decade, an effort has been made to handle uncertainty in information flows in several ways [17]: we can distinguish the contributions as authors consider the uncertainty at the data source level, at the complex events description level or at the output level. Fuzzy logic [18] [19] has been specifically designed to mathematically represent uncertainty and vagueness and can be helpful to address the latter three levels of uncertainty. Our research aims at developing a general-purpose online fuzzy expert system which can handle data or event streams. The goal is to enable human experts to author rules with complex fuzzy relations, to recognize scenarios, to filter data, etc. In fuzzy logic, those relations are words which hide a more or less complex mathematical processing. In this paper, we assume that:

- sensors give correct timestamps: the fuzziness is thus not necessary on timestamps but vagueness is useful to describe the possible relations between them;

- the different values from the sensors are fuzzified in order to both manipulate linguistic terms and handle their inaccuracy.

As an adaptation to fuzzy reasoning, the thirteen Allen's relations have been fuzzified in different ways [20] [21] [8]. Nevertheless, those relations are not sufficient enough in an online system since they rely on an interval algebra whereas an online system can consider partial intervals. We present in this article our work on fuzzy temporal operators for online fuzzy expert systems. It aims at defining simple temporal operators and then at combining them in order to build more complex temporal operators with an intuitive semantics. Such operators are convenient in many applications such as medical monitoring, security with critical scenario recognition, or systems maintenance. 
The paper is organized as follows: in the next section, we discuss related work. Then, section 3 defines the notion of scope, the notations and the examples. Section 4 presents a few base operators which are evaluated on the signal of fuzzy past values. Section 5 describes more complex operators. Finally, section 6 points out the conclusions.

\section{Related work}

This section briefly reviews previous work on bringing temporal relations to fuzzy expert systems. We voluntary prune here all the papers about representation of imprecise or uncertain temporal knowledge as in [5] [6] [7] [8]. Dubois et al [8] also propose some reasoning methods about time based on Zadeh possibility theory. The first attempt to merge both numeric and temporal reasoning at the same time is introduced by Qian [22]: he uses a pattern matching algorithm based on a grid search and defines temporal operators (after, before, ...) which take into account the value of the numeric fuzzy propositions. In [23], the authors propose a new definition of time in fuzzy logic: they define time dependent fuzzy variables, fuzzy sets and fuzzy rules. It consists in considering a time parameter in those objects, for example by changing the membership function of the sets.

Barro et al [24] applied the concept of fuzzy time in medical applications and define a representation language of fuzzy temporal rules (FTR). From this language, Cariñena et al $[25,26]$ propose formulations to compute the value of the FTR for two main propositions called persistence and non-persistence. The propositions applied on a fuzzy interval $T$ defined by means of a possibility distribution $\mu_{T}$ over the time axis $\tau$. Let $\operatorname{DOF}(P, t)$ be the degree of fulfillment of a fuzzy proposition $P$ "Input I is A" at time $t$ :

$$
c D O F(P, t)=\mu_{A}(I(t)) \forall t \in \tau .
$$

The values of signal $I(t)$ are acquired at a constant rate. The two temporal propositions are then defined by [25]:

- Persistence:

$$
c D O F=\bigwedge_{t_{i} \in \tau} D O F\left(P, t_{i}\right) \vee\left(1-\mu_{T}\left(t_{i}\right)\right),
$$

- Non-persistence:

$$
c D O F=\bigvee_{t_{i} \in \tau} \operatorname{DOF}\left(P, t_{i}\right) \wedge \mu_{T}\left(t_{i}\right)
$$

For instance, a persistence proposition may be "temperature was high throughout the last 30 minutes" and a non-persistence proposition may be "temperature was high in the last 30 minutes". Conjointly with Zadeh linguistic quantification, FTR have been successfully applied in robotics [27], people detection [28]. 


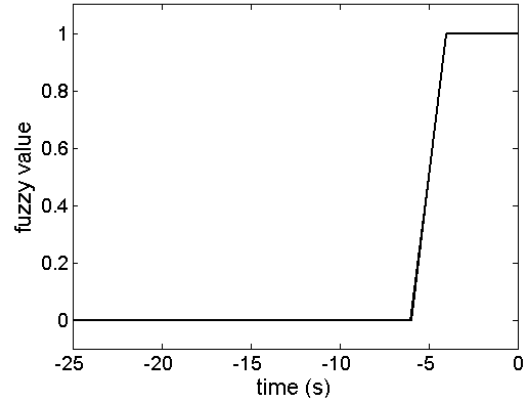

(a) "the last 5 seconds"

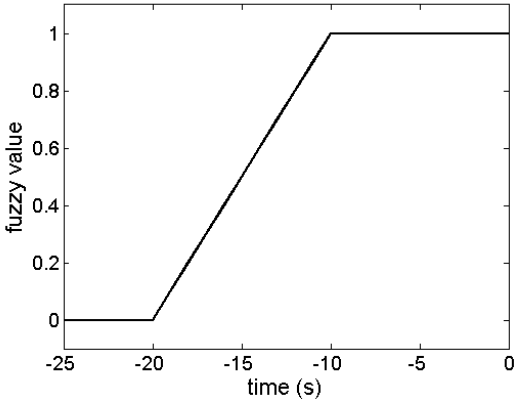

(b) "the last 20 seconds"

Figure 1: Membership functions representing two different temporal scopes.

In [7], the authors describe much more domains of application, such as trend analysis in economy time series, business intelligence, etc.

Our work starts from [25] and the definition of the non-persistence operator. We focus on defining complex temporal relations by combining operators regarding their intuitive definition. To the best of our knowledge, such relations are not available in commercial or open source softwares like Matlab, FuzzyTech, FIS pro, etc.

\section{General considerations}

\subsection{Temporal scope}

To be efficiently implemented, the fuzzy temporal operators described in this paper are applied on operands which are fuzzy expressions, regarding a certain temporal scope. A scope is a range over the past times which terminates at the present moment. In fuzzy logic, a scope is a temporal fuzzy set [25] whose membership function is used to weight the different past values and to define a vague notion of past, for example "recently" or the "last 5 seconds". Theoretically, the scope may be infinite, but for the sake of performance, it has to be finite. In practice, the membership function has a multiline shape, often semi-trapezoidal as shown in figure 1 .

In terms of implementation, it is possible to determine a piecewise sampling rate according to the shape of both signal and membership function to guarantee a good precision of the operators assessment : on a portion of the signal, if both the signal and the membership function are constant, then a low sampling rate is sufficient to guarantee a good accuracy in the computation of the operators.

\section{$3.2 \quad$ Notations}

In the remainder of this paper, we will use the following notations. 
Let $S$ denote a temporal scope. As $S$ is always relative to the present $t_{\text {now }}$, let $\mu_{S}\left(t-t_{\text {now }}\right)$ be the value of the membership function of $S$ for time $t$ : to facilitate the reading, we will use the notation $\mu_{S}(t)$. $S$ always contains the value at time $t_{\text {now }}$, and we denote $S^{*}$ the scope $S-\left\{t_{\text {now }}\right\}$. Supp $(S)$ denotes the support of $S$.

Operators $\bigwedge$ and $\bigvee$ stand respectively for a t-norm and a t-conorm: without loss of generality, the examples are given with Zadeh's t-norm and t-conorm.

Finally, let $E, E_{1}$ and $E_{2}$ be fuzzy-valued expressions. eval $(E, t)$ (respectively $E_{1}$, or $E_{2}$ ) be the value of the operand expression at time $t$. Typically, $\forall t_{i} \in S, \operatorname{eval}\left(E, t_{i}\right)$ is the signal of the past values of $E$ regarding the support of the scope $S$. When the expression is a simple expression like "the temperature is high", it is evaluated by $\operatorname{eval}\left(E, t_{i}\right)=\mu_{h i g h}\left(E\left(t_{i}\right)\right)$. For the sake of simplicity, we will omit in the notations to cite inputs on which the expression depends.

\subsection{Examples}

Figure 2a shows an example of signal for a temperature measurement. Regarding the fuzzy set "high" (figure $2 b$ ), figure $2 c$ shows the signal for the proposition "the temperature is high". The temperature signal has been chosen in order to illustrate the usefulness and the behavior of temporal operators proposed in this paper. This signal can be split into two parts:

- from the 5 th to the 30 th second, it describes cycles of high temperatures ;

- from the 40th to the 55 th second, it describes a steady high temperature state.

Consequently, the fuzzy expression "the temperature is high" catches these particular patterns with very high fuzzy values, and decreases to 0 between these two parts during a little bit less than 10 seconds.

Figure 3 shows another fuzzy proposition "the rainfall is high" that will be used in the remainder of this article. Note that both signals have a constant sampling rate of 1 second over 1 minute. After one minute, no more inputs are received from the sensors since the values are constant: this behavior is typical of event streams.

\section{Base operators}

In this section, we describe the base operators which will be combined later into new operators.

\subsection{Occurrence}

The first fuzzy temporal operator is unary and indicates whether a certain phenomenon has occurred regarding a certain scope. Here a phenomenon is the activation of a fuzzy expression operand, like a fuzzy proposition "temperature 
is high", a conjunction "temperature is high and luminosity is high", or any fuzzy-valued expression. This operator can be reformulated as: in at least one moment of the scope, the operand has a non-null fuzzy value. It matches the specifications of the existential quantifier in [7] or the non-persistence relation in [25]: it consists in the disjunction over all the moments $t_{i}$ of the scope of the conjunction between the value of the operand at time $t_{i}$ weighted by the membership function.

The temporal relation "occurrence", for instance "E has occurred during S", can be written:

$$
\operatorname{lOcc}\left(E, S, t_{\text {now }}\right)=\bigvee_{t \in \operatorname{supp}(S)} \operatorname{eval}(E, t) \wedge \mu_{S}(t)
$$

Figure 4 shows the application of the $O c c$ operator to the expression "the temperature is high" regarding both temporal scopes shown in figure 1 . Whereas both curves raise identically at the beginning, the expression evaluated with the shortest scope decreases gradually to zero from the 34th to the 39th second: indeed, fuzzy values become lower and lower while older and older. On the contrary, with the largest scope, the output signal of the occurrence expression is smoother, hiding the gap obtained before.

\subsection{Ratio operator}

In the terminology of Cariñena [25], we introduce a reduction operator called "ratio" which aggregates the different activations of the operand expression $E$ over a scope $S$. The result is then divided by a value corresponding to the sum of 1 over the same scope. The Ratio operator indicates how much an expression has been true over a scope. It can be formulated by:

$$
\operatorname{lRatio}\left(E, S, t_{\text {now }}\right)=\frac{\int_{t \in \operatorname{supp}(S)} \operatorname{eval}(E, t) \wedge \mu_{S}(t)}{\int_{t \in \operatorname{supp}(S)} \mu_{S}(t)}
$$

where $\int_{t \in \operatorname{supp}(S)} \operatorname{eval}(E, t) \wedge \mu_{S}(t)$ is the area under the signal composed of the past values of $E$ regarding $S$ and $\int_{t \in \operatorname{supp}(S)} \mu_{S}(t)$ is the area under the membership function of $S$. This operator is simply Zadeh's relative count applied to a temporal domain [29].

The figure 5 shows the application of the Ratio operator to the proposition "the temperature is high" with both temporal scopes. As this operator is built on an integral, it shows how it smoothes the input signal. The larger the scope, the smoother the curve : larger scopes weaken the resulting signal slopes.

\subsection{Expiration}

These two base operators have a particularity when applied on a finite scope. Since our system also has to deal with event streams or data streams with 
dynamic sampling, inputs may not change for a while. Meanwhile, time goes by and the value of expressions containing Occ or Ratio operators may change. Their values can thus expire, that is to say that they are valid only for a while. As the scope has a finite duration and is anchored on $t_{n o w}$, they must be reassessed. The delay of expiration is customizable to fit the application of these operators. For instance, if events arrive every 15 minutes, it is not necessary to make them expire every second. This is a particular feature of our online fuzzy expert system: the value of the outputs can change even if inputs have not changed.

The expiration of these operators is triggered regarding different criteria:

- The occurrence operator keeps expiring as long as its value is strictly greater than 0 . Indeed, if it reaches 0 and if operands do not change anymore, the occurrence will keep a null value, so it can stop expiring.

- The ratio operator $\operatorname{Ratio}\left(E, t_{n o w}, S\right)$ keeps expiring if, at time $t_{n o w}$ :

- the signal associated with the scope $S$ contains only one value;

- $\operatorname{Ratio}\left(E, S, t_{\text {now }}\right)$ is null but $\operatorname{eval}\left(E, t_{\text {now }}\right)>0$.

The effect of the expiration can be seen on figure 4 or on figure 5 : the new expressions continue to be evaluated after one minute while no more input data arrive.

\section{Advanced operators}

This section aims at showing how the previous operators can be combined into more complex temporal relations which can be conveniently used by end-users. In the previous section, we claimed the operand expression $E$ can be any fuzzyvalued expression, including temporal expressions. The advantage is that both the computation and the implementation of the expiration are handled by the base operators, so that the implementation of new operators is straightforward.

We present further a few examples of advanced fuzzy temporal operators which are convenient to characterize the persistence, the precedence or the cyclicity of the values of fuzzy expressions.

\subsection{Persistence}

The persistence operator has been described in section 2. We propose in this article another formalization based on the "Occ" operator. Indeed, the persistence of a phenomenon throughout a given scope is the fact that at each moment of the scope, the phenomenon is observed. In other words, for a given operand expression $E$, its negation must not have occurred:

$$
l \operatorname{Pers}\left(E, S, t_{\text {now }}\right)=\neg O c c\left(\neg E, S, t_{\text {now }}\right) .
$$


For a given De Morgan triplet (i.e. a t-norm, a t-conorm and a fuzzy negation which respect De Morgan laws), expression 6 is equivalent to expression 2 (the proof is straightforward).

Figure 6 shows the application of the Pers operator to the fuzzy expression "the temperature is high" regarding both temporal scopes shown in figure 1. We can see that a persistence expression reaches its maximum value only when the steady-state of high temperatures is itself reached, with a small delay.

This operator can perform only on signals with at least two samples to ensure whether the expression persists or not. Thus, it is possible to observe a small delay before the first evaluation of this operator.

We can observe the following relations between the operators:

$$
\begin{gathered}
c \operatorname{Pers}\left(E, S, t_{\text {now }}\right) \leq \operatorname{eval}\left(E, t_{\text {now }}\right) \leq \operatorname{Occ}\left(E, S, t_{\text {now }}\right) \\
\operatorname{Pers}\left(E, S, t_{\text {now }}\right) \leq \operatorname{Ratio}\left(E, S, t_{\text {now }}\right) \leq \operatorname{Occ}\left(E, S, t_{\text {now }}\right) .
\end{gathered}
$$

With the Occ and Pers operators, we can investigate if a phenomenon has persisted for a while during the scope: for instance, " $E$ has persisted 5 seconds during the last hour". Two scopes are involved in this relation: the first one characterizes the duration of the persistence, the second one the duration of the global relation. This kind of temporal relation can be very useful for system diagnosis and maintenance for instance.

The idea is to compute the persistence of a phenomenon on a scope $S_{\text {duration }}$, the last " 5 seconds" in the example above. We then just have to look for the occurrences of such a persistence:

$$
\begin{gathered}
l \operatorname{PersWhile}\left(E, S_{\text {duration }}, S, t_{\text {now }}\right)= \\
\operatorname{Occ}\left(\operatorname{Pers}\left(E, S_{\text {duration }}, t_{\text {now }}\right), S, t_{\text {now }}\right) .
\end{gathered}
$$

By definition of the persistence operator, if the phenomenon disappears during $S_{\text {duration }}$, the PersWhile relation is null. It is possible to be more tolerant by replacing the persistence by the Ratio operator:

$$
\begin{aligned}
& \text { lPersWhileTol }\left(E, S_{\text {duration }}, S, t_{\text {now }}\right)= \\
& \operatorname{Occ}\left(\operatorname{Ratio}\left(E, S_{\text {duration }}, t_{\text {now }}\right), S, t_{\text {now }}\right) .
\end{aligned}
$$

Figures 7 and 8 show respectively these two last operators applied to the proposition "the temperature is high" for a duration of 5 seconds during the last 20 seconds. Even if the tolerant expression PersWhileTol has higher values than the strict one (PersWhile), both of them reach the maximum value of 1 only when high temperatures are persistent, with a small delay.

\subsection{Precedence}

The precedence is the fact that a phenomenon $f$ before a second one. This is thus a binary operator which takes two fuzzy expressions as operands. 
As a first attempt to formalize a fuzzy precedence operator from the base operators, we define the relation " $E_{1}$ started before $E_{2}$ " by:

$$
\begin{array}{r}
l \operatorname{StartBef}\left(E_{1}, E_{2}, S, t_{\text {now }}\right)= \\
\operatorname{Occ}\left(E_{2} \wedge \operatorname{Occ}\left(E_{1} \wedge \neg E_{2}, S, t_{\text {now }}\right), S^{*}, t_{\text {now }}\right) .
\end{array}
$$

The deepest $O c c$ operator in equation 10 indicates whether in the scope there exists a moment when the phenomenon $E_{1}$ was observed while the phenomenon $E_{2}$ was not. The precedence is true if this $O c c$ is true and if $E_{2}$ is observed. The other $O c c$ operator in equation 10 looks for such a moment in the scope.

We can define a stronger precedence relation with a "before" operator. The semantic of the relation "before" has been defined in [9] and has been used in many papers: to Allen, " $E_{1}$ precedes $E_{2}$ " is true if $E_{1}$ has started and has stopped before $E_{2}$ began. The StartBef relation above does not match the semantics of Allen's precedence. To define the value of a " $E_{1}$ before $E_{2}$ " relation, $E_{1}$ must have started before $E_{2}$, and $E_{1}$ must not be observed anymore while $E_{2}$ must have been observed:

$$
\begin{array}{r}
\operatorname{lBef}\left(E_{1}, E_{2}, S, t_{\text {now }}\right)= \\
\operatorname{StartBef}\left(E_{1}, E_{2}, S^{*}, t_{\text {now }}\right) \\
\wedge \neg \operatorname{eval}\left(E_{1}, t_{\text {now }}\right) \wedge \operatorname{Occ}\left(E_{2}, S, t_{\text {now }}\right) .
\end{array}
$$

Figures 9 and 10 show respectively the application of the StartBef and the $B e f$ operators to the two expressions $E_{1}$ "rainfall is high" and $E_{2}$ "temperature is high". In the case of the StartBef relation, the values are high at the beginning and decrease regularly after the expression "the rainfall is high" is not true anymore. Considering a larger scope enables to maintain the activation of the precedence relation longer. Nesting the $O c c$ operator prolongs the scope duration. Figure 10 shows that the value of operator is different from 0 until $E_{1}$ is not observed anymore in the scope.

\subsection{Periodicity}

Finally, it may be interresting to be able to express the fact that a phenomenon should be observed regularly during a certain period of time (defined by a scope $\left.S_{\text {period }}\right)$, for instance, it has to happen every hour of the last day. The assessment of the regularity fails as soon as the occurence of the phenomenon has not been observed on the scope $S_{\text {period }}$ :

$$
\begin{array}{r}
\operatorname{lOccEvery}\left(E, S_{\text {period }}, S, t_{\text {now }}\right)= \\
\operatorname{Pers}\left(\operatorname{Occ}\left(E, S_{\text {period }}, t_{\text {now }}\right), S, t_{\text {now }}\right) .
\end{array}
$$

The figure 11 shows the expression "the temperature is high every 5 seconds during 20 seconds". The maximum value of this expression is reached at

least once for both high temperature areas ([20-30s], 40s) but decreases to zero between them because of the low temperature area. 


\section{Conclusion}

In this article, we introduced a compositional paradigm to build temporal operators. Two main base operators, "occurrence" and "ratio", found in the literature, are combined into new more complex temporal operators. Those operators may help experts to characterize the persistence, the precedence or the periodicity of events and data represented by fuzzy expressions. Online fuzzy expert systems have a role to play in data and event streams processing, bringing the ability to describe with words phenomena and scenarios, while hiding the mathematical formalizations. These fuzzy temporal operators indicate in one fuzzy value both the activation of the operands and their temporal layout.

We also define the concept of expiration of an expression: even if the inputs have not changed, an "expirable" expression indicates it needs to be evaluated again. Thus, an online fuzzy expert system is able to change the outputs even if no inputs changed. Expiration is a convenient feature to process event streams.

The implemented system can be used in several applications. For instance, in medical monitoring, it may be useful to represent the persistence of the symptoms, or their temporal adjustment. In technical diagnostics, persistence and periodicity can characterize the criticality of breakdowns. With the potential of combinations, more temporal relations may be defined.

\section{Acknowledgment}

We want to thank Tristan Galliez and Etienne Petrel for helping us implementing the operators and the demonstration.

\section{References}

[1] G. Cugola and A. Margara, "Processing flows of information: From data stream to complex event processing," ACM Comput. Surv., vol. 44, no. 3, pp. 15:1-15:62, Jun. 2012.

[2] D. Wang, E. A. Rundensteiner, and R. T. Ellison, III, "Active complex event processing over event streams," Proc. VLDB Endow., vol. 4, no. 10, pp. 634-645, Jul. 2011.

[3] C. Dousson, "Extending and unifying chronicle representation with event counters." in ECAI, F. van Harmelen, Ed. IOS Press, 2002, pp. 257-261.

[4] A. Artikis, A. Skarlatidis, F. Portet, and G. Paliouras, "Logic-based event recognition," Knowledge Engineering Review, vol. 27, no. 4, pp. 469-506, 2012.

[5] K. Walzer, M. Groch, and T. Breddin, "Time to the rescue - supporting temporal reasoning in the rete algorithm for complex event processing," in Database and Expert Systems Applications, ser. Lecture Notes in Computer Science. Springer Berlin Heidelberg, 2008, vol. 5181, pp. 635-642. 
[6] S. Schockaert, M. D. Cock, and E. Kerre, Reasoning About Fuzzy Temporal and Spatial Information from the Web, ser. Intelligent Information Systems. World Scientific, 2010, vol. 3.

[7] S. Barro, A. Bugarín, P. Cariñena, F. Díaz-Hermida, and M. Mucientes, "Fuzzy temporal rule-based systems: New challenges," in Actas del XIV Congreso Español sobre Tecnologías y Lógica Fuzzy (ESTYLF), Langreo (Spain), 2008, pp. 507-514.

[8] D. Dubois, A. Hadj Ali, and H. Prade, "Fuzziness and uncertainty in temporal reasoning," Journal of Universal Computer Science, vol. 9, no. 9, pp. 1168-1194, 2003.

[9] J. F. Allen, "Maintaining knowledge about temporal intervals," Communications of the ACM, vol. 26, no. 11, pp. 832-843, Nov. 1983.

[10] J. A. dos Santos, C. Braga, D. C. Muchaluat-Saade, C. Roisin, and N. Layaïda, "Spatio-temporal validation of multimedia documents," in Proceedings of the 2015 ACM Symposium on Document Engineering, ser. DocEng '15. New York, NY, USA: ACM, 2015, pp. 133-142.

[11] T. Watanabe, "A proposal of smart means to retrieve impressively-referred document-page," in Proceedings of the 9th International Conference on Ubiquitous Information Management and Communication, ser. IMCOM '15. New York, NY, USA: ACM, 2015, pp. 83:1-83:8.

[12] A. Cimatti, A. Micheli, and M. Roveri, "An smt-based approach to weak controllability for disjunctive temporal problems with uncertainty," Artificial Intelligence, vol. 224, pp. 1 - 27, 2015.

[13] C. Dousson and P. L. Maigat, "Chronicle recognition improvement using temporal focusing and hierarchisation," in Proceedings of IJCAI, 2007, pp. 324-329.

[14] C. Choppy, O. Bertrand, and P. Carle, "Coloured petri nets for chronicle recognition," in Reliable Software Technologies, Ada-Europe 2009, ser. Lecture Notes in Computer Science, F. Kordon and Y. Kermarrec, Eds. Springer Berlin Heidelberg, 2009, vol. 5570, pp. 266-281.

[15] K.-U. Schmidt, R. Stühmer, and L. Stojanovic, "Blending complex event processing with the rete algorithm," in iCEP2008: 1st International workshop on Complex Event Processing for the Future Internet colocated with the Future Internet Symposium (FIS2008), D. Anicic, C. Brelage, O. Etzion, and N. Stojanovic, Eds., vol. Vol-412. CEUR Workshop Proceedings, September 2008.

[16] A. Artikis, C. Baber, P. Bizarro, C. Canudas-de Wit, O. Etzion, F. Fournier, P. Goulart, A. Howes, J. Lygeros, G. Paliouras, A. Schuster, and I. Sharfman, "Scalable proactive event-driven decision making," 
Technology and Society Magazine, IEEE, vol. 33, no. 3, pp. 35-41, Fall 2014.

[17] E. Alevizos, A. Skarlatidis, A. Artikis, and G. Paliouras, "Complex event processing under uncertainty: A short survey," in Proceedings of the Workshops of the EDBT/ICDT 2015 Joint Conference, ser. CEUR Workshop Proceedings, P. M. Fischer, G. Alonso, M. Arenas, and F. Geerts, Eds., vol. 1330, 2015, pp. 97-103.

[18] L. Zadeh, "Fuzzy sets," Information and Control, vol. 8, no. 3, pp. 338 $353,1965$.

[19] L. A. Zadeh, "The concept of a linguistic variable and its application to approximate reasoning," Information Sciences, vol. 9, pp. 43-80, 1975.

[20] N. A. A. Manaf and M. R. Beikzadeh, "Crisp-fuzzy representation of allen's temporal logic," in Proceedings of the 25th Conference on Proceedings of the 25th IASTED International Multi-Conference: Artificial Intelligence and Applications, ser. AIAP'07. Anaheim, CA, USA: ACTA Press, 2007, pp. $174-179$.

[21] S. Schockaert, M. De Cock, and E. E. Kerre, "Fuzzifying allen's temporal interval relations," Trans. Fuz Sys., vol. 16, no. 2, pp. 517-533, Apr. 2008.

[22] D. G. Qian, "Representation and use of imprecise temporal knowledge in dynamic systems," Fuzzy Sets and Systems, pp. 59-77, 1992.

[23] J. Virant and N. Zimic, "Attention to time in fuzzy logic," Fuzzy Sets and Systems, vol. 82, no. 1, pp. 39 - 49, 1996.

[24] S. Barro, R. Marin, J. Mira, and A. R. Patan, "A model and a language for the fuzzy representation and handling of time," Fuzzy Sets and Systems, vol. 61, no. 2, pp. 153 - 175, 1994.

[25] P. Cariñena, A. Bugarín, M. Mucientes, and S. Barro, "A language for expressing fuzzy temporal rules," Mathware and Soft Computing, vol. 7, no. 2-3, pp. 213-227, 2000.

[26] P. Cariñena, A. Bugarín, M. Mucientes, F. Díaz-Hermida, and S. Barro, Technologies for Constructing Intelligent Systems 2: Tools, ser. Studies in Fuzziness and Soft Computing. Physica-Verlag, 2002, vol. 90, ch. Fuzzy Temporal Rules: A Rule-based Approach for Fuzzy Temporal Knowledge Representation and Reasoning, pp. 237-250.

[27] M. Mucientes, R. Iglesias, C. Regueiro, A. Bugarín, P. Cariñena, and S. Barro, "Use of fuzzy temporal rules for avoidance of moving obstacles in mobile robotics," in Proceedings of the Eusflat-Estylf Joint Conference, Palma de Mallorca (Spain), 1999, pp. 167-170. 
[28] M. Mucientes and A. Bugarín, "People detection with quantified fuzzy temporal rules," in Proceedings of the IEEE International Conference on Fuzzy Systems (FUZZ-IEEE), London (UK), 2007, pp. 1149-1154.

[29] L. Zadeh, "A computational approach to fuzzy quantifiers in natural languages," Computers and Mathematics, vol. 9, pp. 149-184, 1983. 


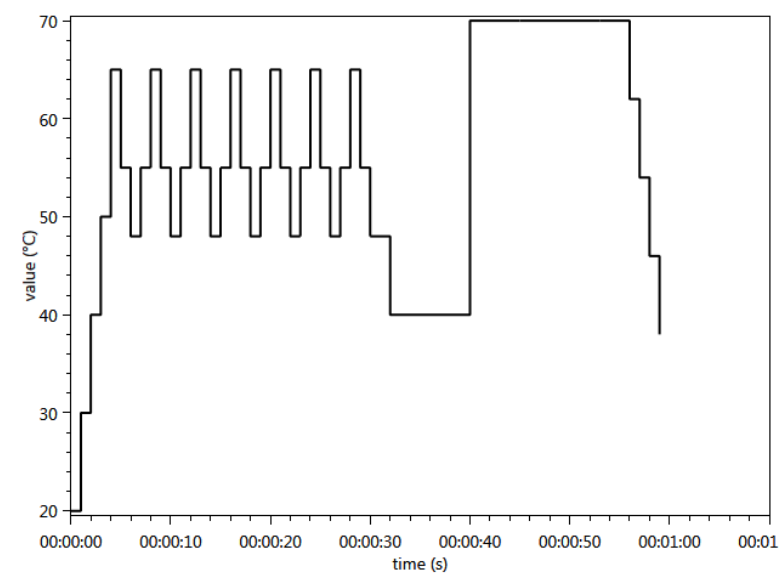

(a) Temperature signal

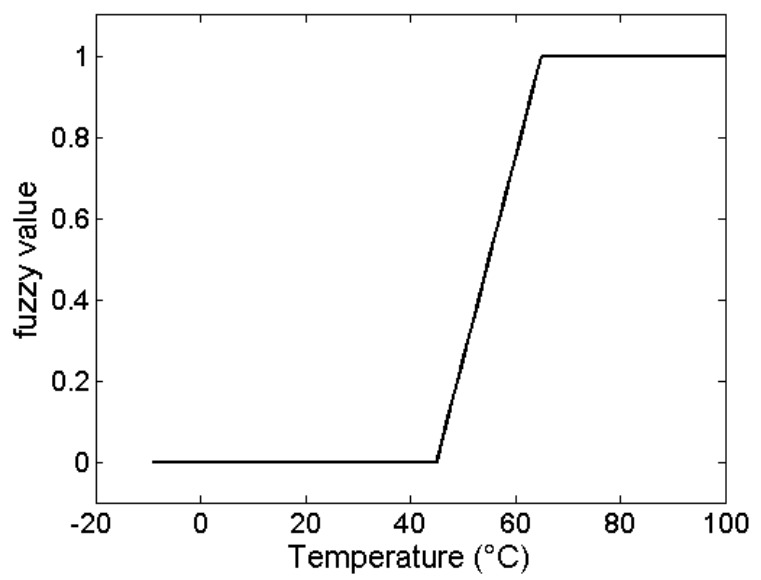

(b) Membership function of the fuzzy set "high" for a temperature

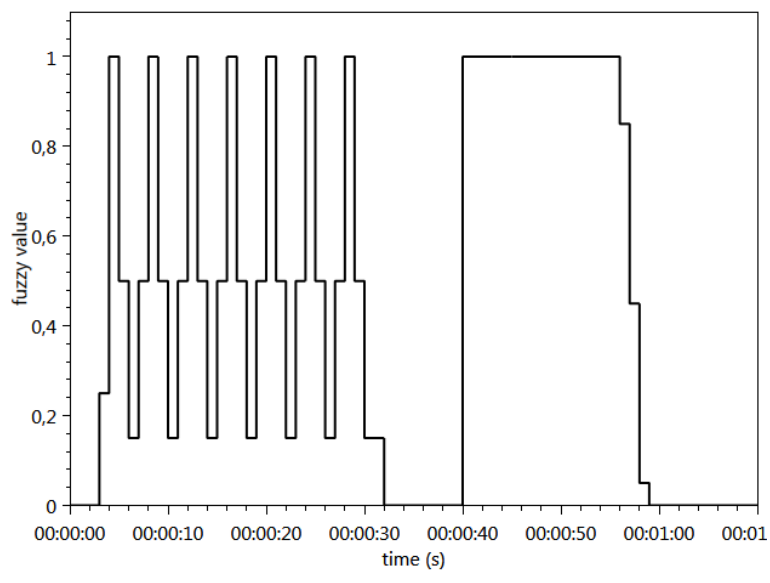

(c) Proposition "the temperature is high" 14

Figure 2: Input signal of a temperature sensor, a membership function associated to its domain, and evaluation of the resulting fuzzy proposition. 


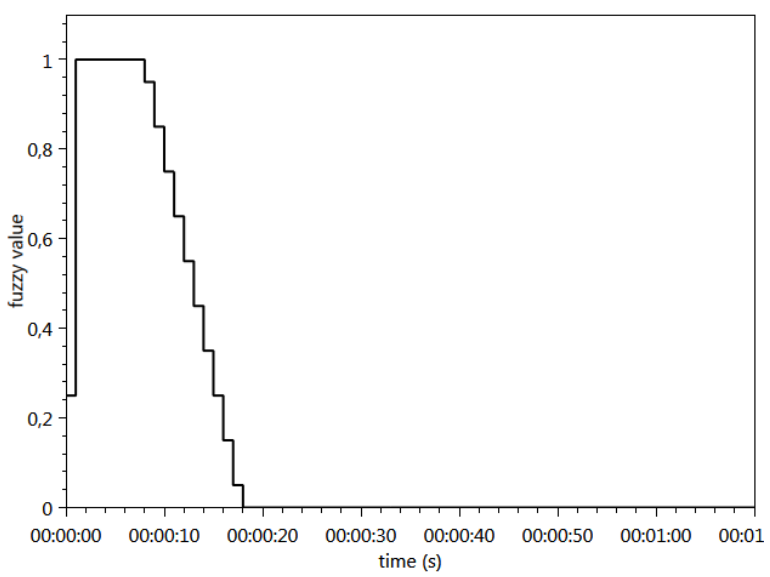

Figure 3: The fuzzy proposition "the rainfall is high".

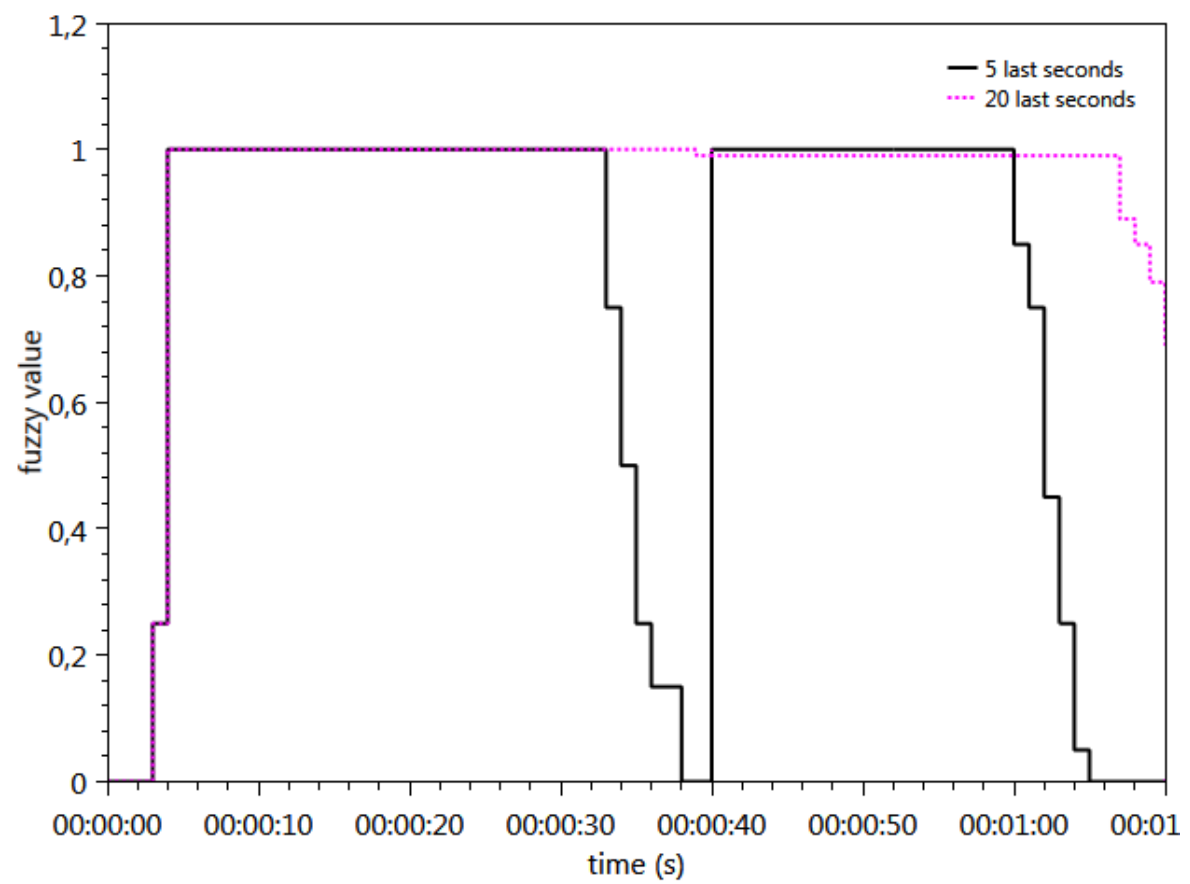

Figure 4: Application of the Occ operator to the expression "the temperature is high" on two different scopes. 


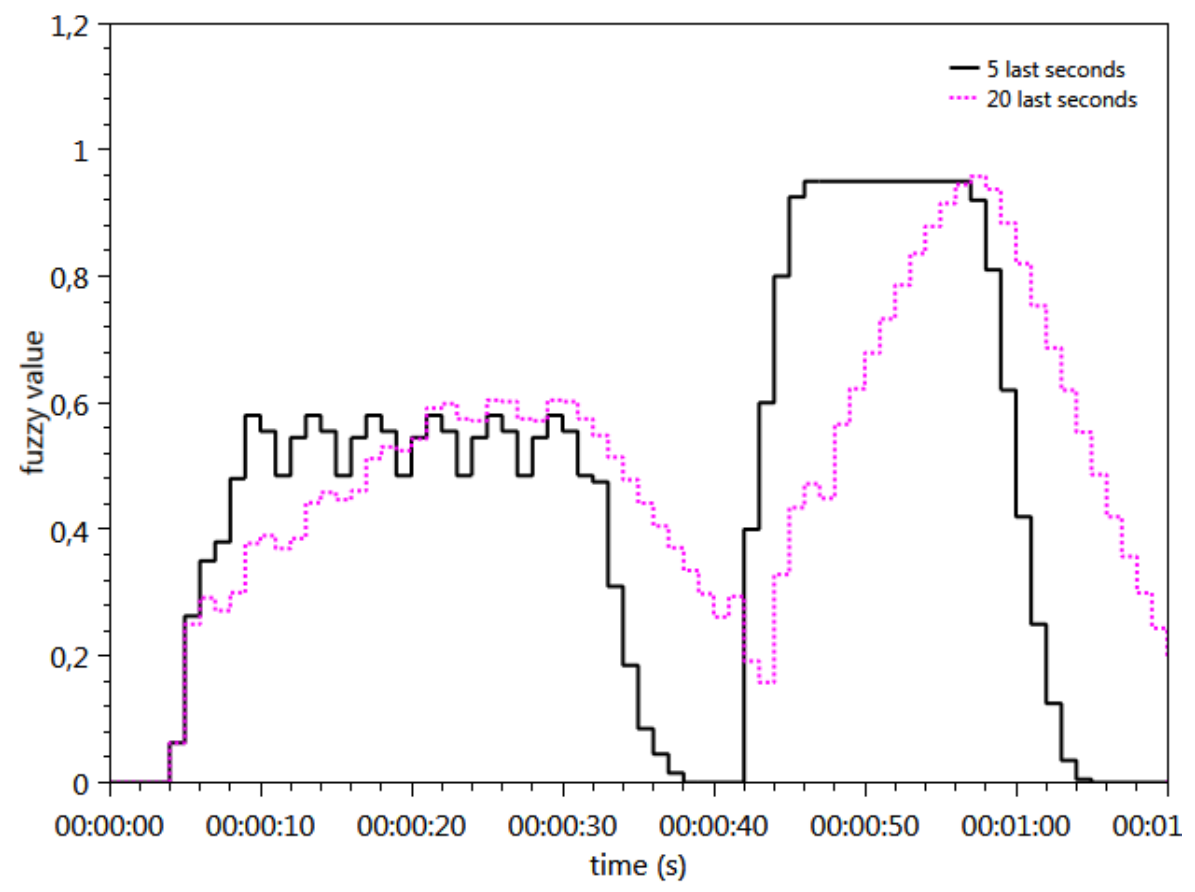

Figure 5: Application of Ratio operator to the expression "the temperature is high" on two different scopes. 


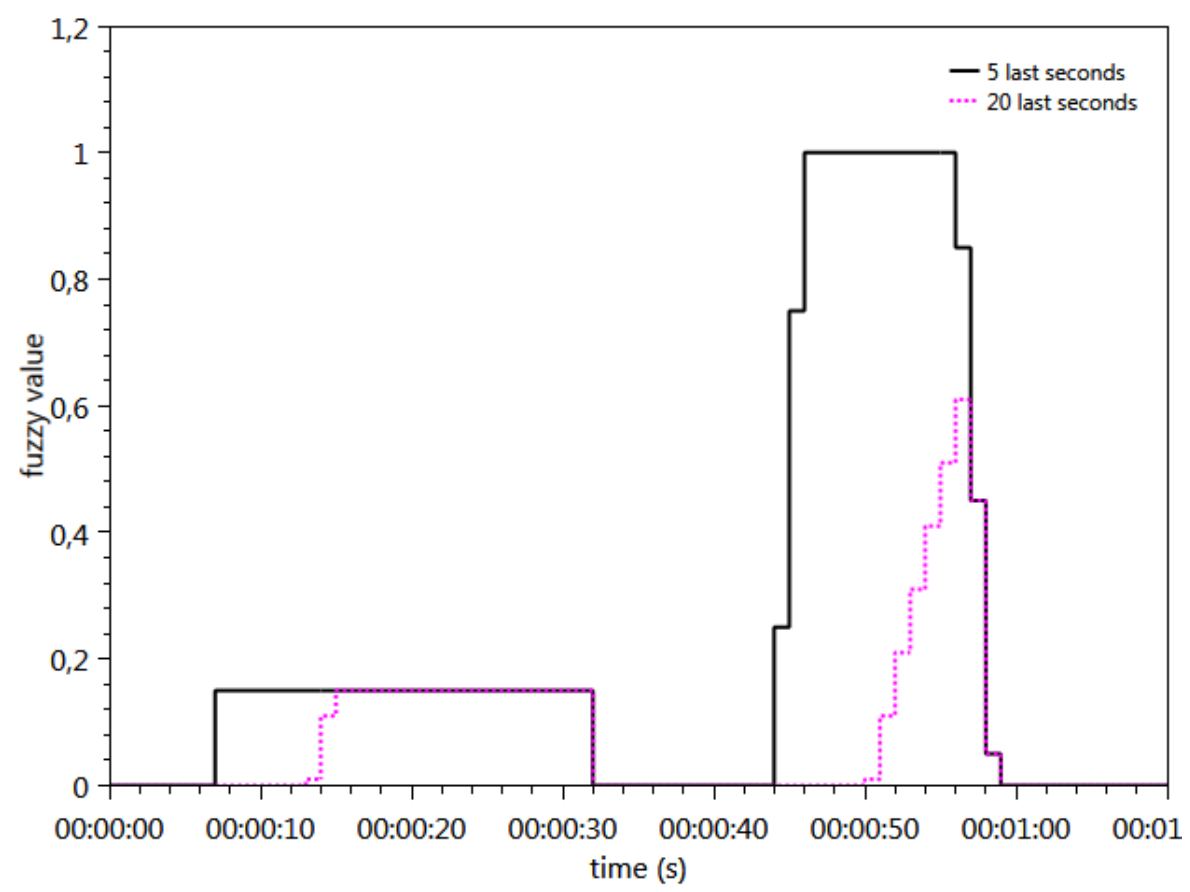

Figure 6: Application of the Pers operator to the expression "the temperature is high" on two different scopes. 


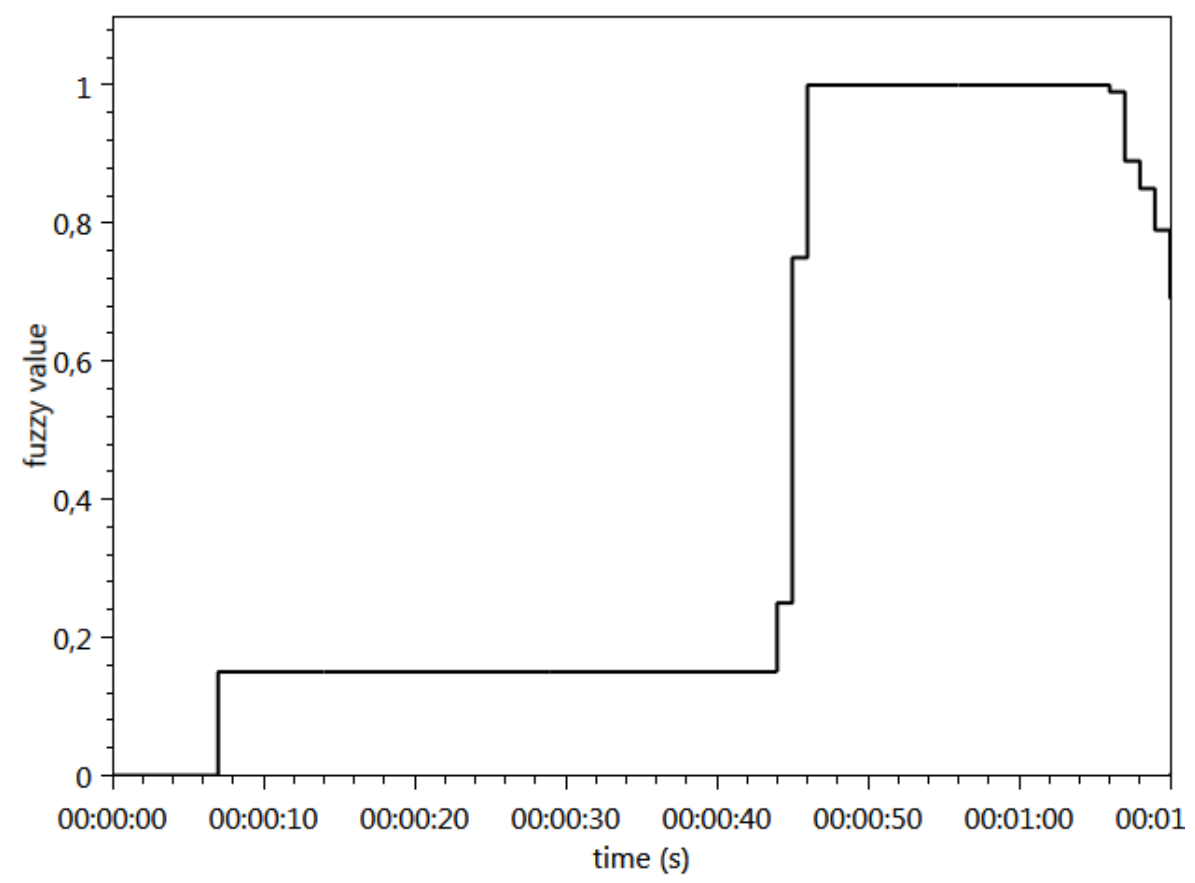

Figure 7: Application of the PersWhile operator to the expression "the temperature is high" with a persistence of 5 seconds during the last 20 seconds. 


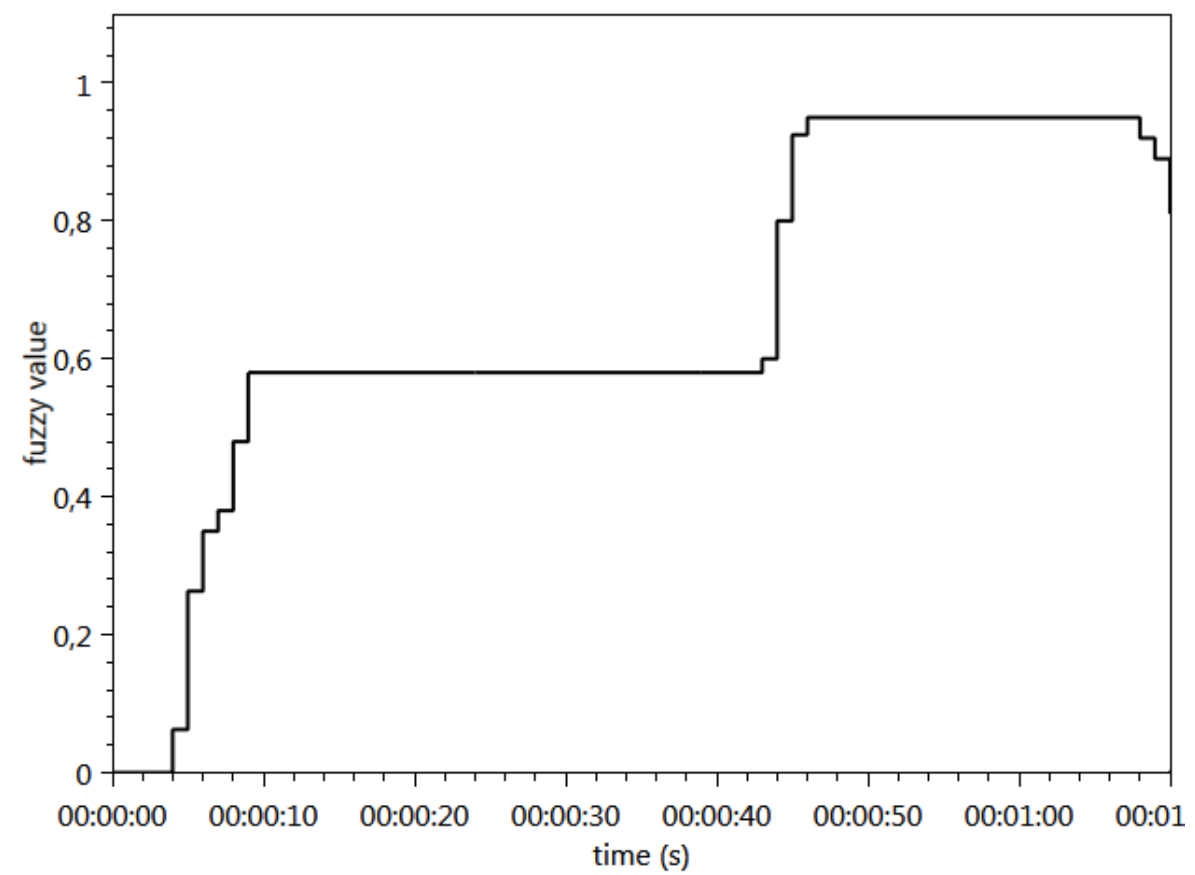

Figure 8: Application of the PersWhileTol operator to the expression "the temperature is high" with a ratio computed on 5 seconds during the last 20 seconds. 


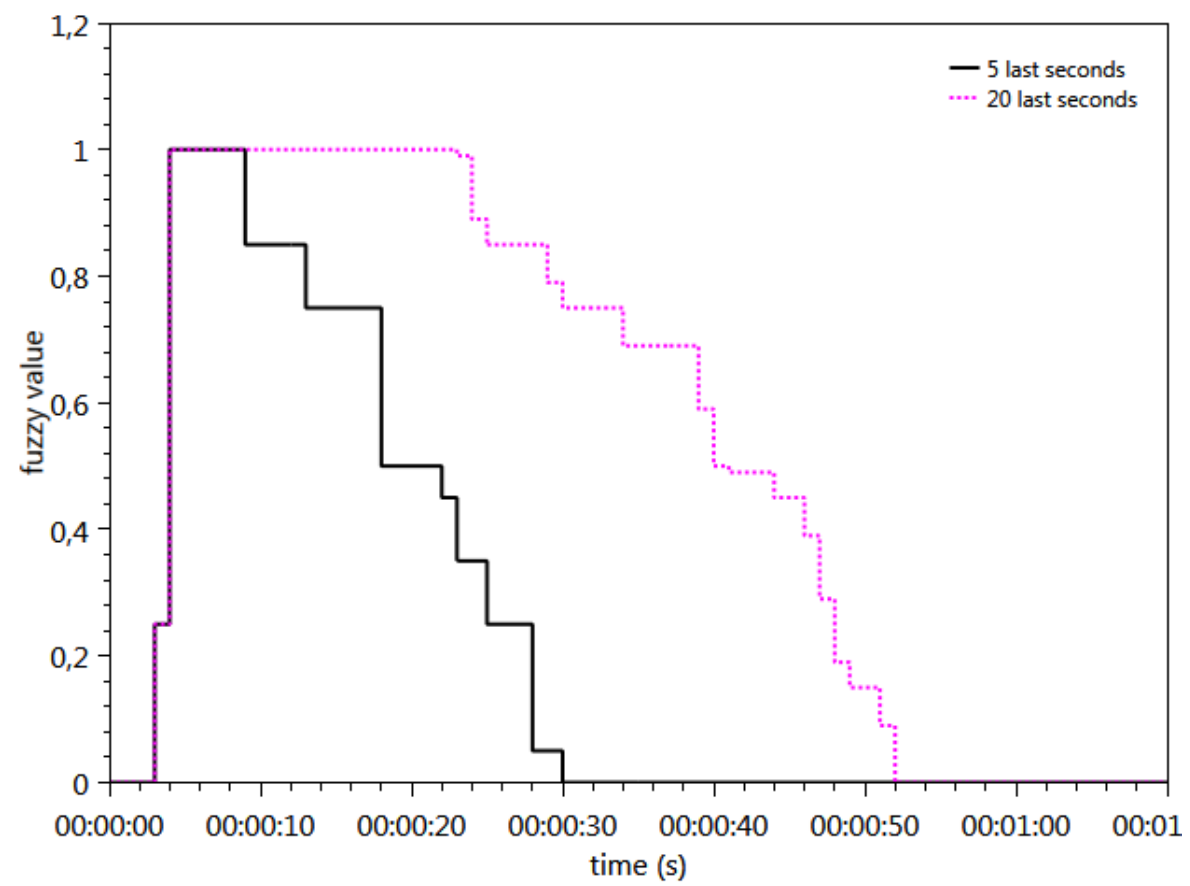

Figure 9: Evaluation of the expression "the rainfall is high has started before that the temperature is high" on two different scopes. 


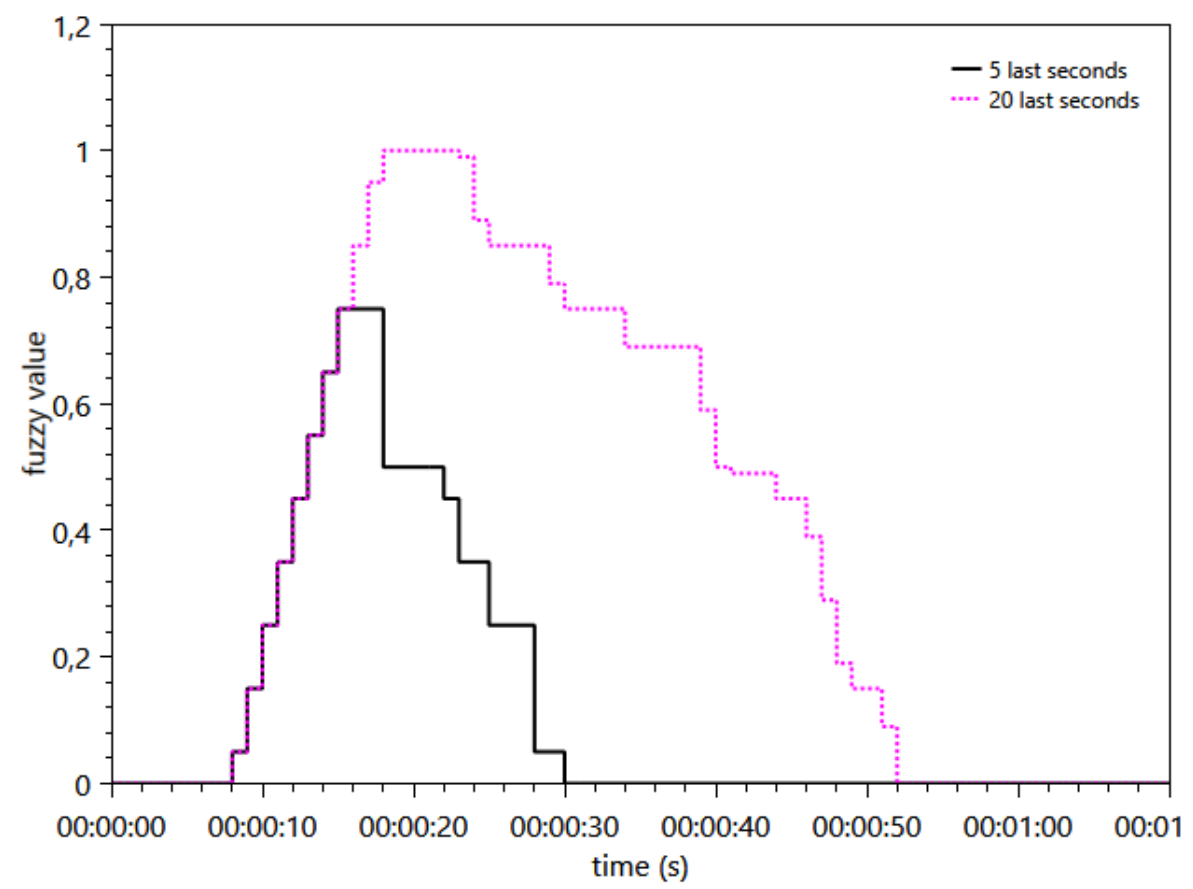

Figure 10: Evaluation of the expression "the rainfall is high before the temperature is high" on two different scopes. 


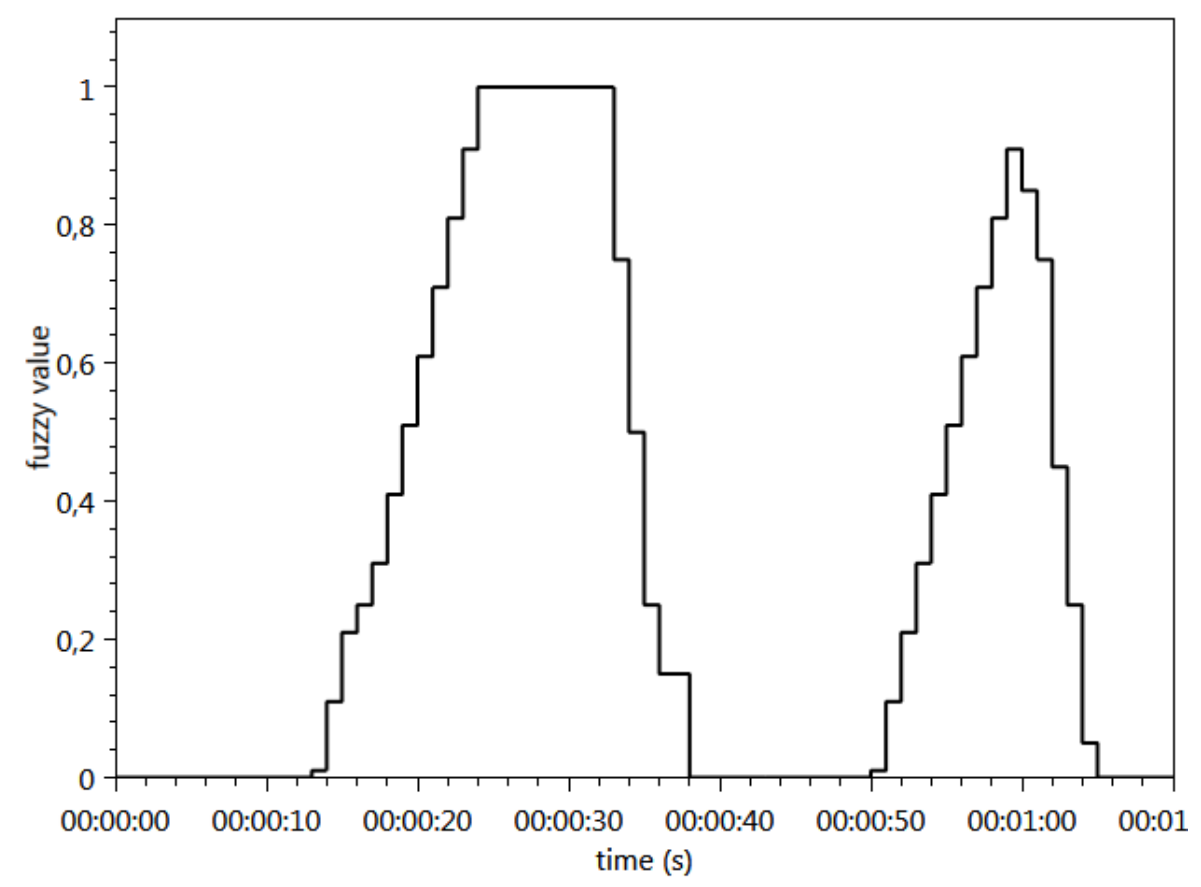

Figure 11: Application of OccEvery operator to the expression "the temperature is high" on two different scopes. 